\title{
Surrogate modeling of waveform response using singular value decomposition and Bayesian optimization
}

\author{
Kohei SHINTANI*, Takao FUJIMOTO*, Masaaki OKAMOTO*, Atsuji ABE* and Yasushi YAMAMOTO* \\ *Toyota Motor Corporation \\ 1,Toyota-cho, Toyota,Aichi,471-8572 Japan \\ E-mail: kohei_shintani@mail.toyota.co.jp
}

Received: 31 March 2020; Revised: 29 June 2020; Accepted: 1 October 2020

\begin{abstract}
In the early stage of vehicle development, it is required to implement a target cascading study by solving inverse problems. However, simulation costs of vehicle dynamics to predict transient responses and frequency responses make the target cascading study difficult. The purpose of this paper is to propose a method to construct a surrogate model which can predict waveform responses and a solution of Bayesian optimization using posterior distribution of trained waveform responses. Replacement of the expensive simulation by the more economical surrogate model can enhance the target cascading study. In this paper, we construct a vectorized training data matrix from the waveform responses which can be evaluated from CAE simulations based on the Design of Experiments. In this proposed method, supervised and unsupervised learning are introduced. The singular value decomposition is used as a feature extraction method (Unsupervised learning) and applied to the training data. Obtained singular vectors are used as feature modes to represent the training data. Gaussian Process model is introduced as a regression model (Supervised learning) and applied to each weight of feature modes which can be obtained by projection of training data to feature modes. The waveform response can be predicted by the superposition of prediction feature values and feature modes. By using the posterior distribution of trained Gaussian Process, Expected Improvement function is evaluated and used in Bayesian optimization to minimize a cost function which is evaluated from the posterior mean of a predicted waveform. The feasibility of the proposed method is illustrated by an application for the suspension design problem of impact harshness phenomenon.
\end{abstract}

Keywords : Gaussian process, Design of experiments, Singular value decomposition, Bayesian optimization, Inverse problem, Vehicle dynamics, Multibody dynamics

\section{Introduction}

In the early stage of vehicle development, it is important to study feasible and robust target values which satisfy all system performances in order to make subsequent processes more efficiently without critical rework. Computer simulation is widely used to implement the target setting study because realistic test models are not always available especially in the early stage of vehicle development. However, simulation costs of multibody dynamics such as ride comfort, handling, and NVH (Noise, Vibration, and Harshness) to predict transient responses and frequency responses make the target cascading study difficult. The purpose of this paper is to propose a method to construct a surrogate model which can predict waveform response and a Bayesian optimization method using the trained surrogate model. Replacement of expensive simulations by more economical surrogate models can enhance application ranges of the target cascading study.

The dynamic behavior of vehicles has been studied in the past century (Gillespie, 1992). With advancements in the performance of a workstation, multibody dynamics simulation has become possible to handle a large degree of complex problem and widely used in the vehicle industry in order to evaluate vehicle dynamics performance. In addition to the computational advancements, model reduction methods such as component mode synthesis (CMS) are widely applied to 
elastic body parts (Hintz, 1975). However, even though the computational cost of the multibody dynamics simulation can be dramatically reduced with these advancements, it still takes hours or days in case evaluation of vehicle dynamics due to high dimensional and high nonlinearity of the problem. This computational burden makes it difficult to study target cascading because numbers of evaluations using expensive simulations are required during the study.

Another approach to reduce the computational burden is to introduce Machine Learning (ML) to replace expensive simulations by surrogate models. The surrogate models are approximation models such as regression models or response surface models that can mimic responses of simulations as close as possible while being computationally much cheaper. There is a wide variety of regression models that can be used as a surrogate model such as Random Forests (Hutter, et al., 2011), Support Vector Machines, Least-Squares Support Vector Machines (Suykens and Vandewalle, 1999), MultiLayer Perceptrons (MLPs) (Zăvoianu, et al., 2013; Zăvoianu, et al., 2016), Bayesian Neural Networks (Fonseca, et al., 2003), Gaussian Processes (GP) (Rasmussen and C. K. Williams, 2006; Gorissen, 2010) and linear regression. In this paper, GP model is introduced for the regression model. GP model is mathematically the same as Kriging model. However, the notation and derivation are done from a completely different perspective in literature. Kriging is derived from Best Linear Unbiased Predictor(BLUP) and frequentist approach, while GP is approached from Bayesian statistics. An advantage of GP model is predictable not only mean response but also prediction interval as shown in Fig.1. By using the posterior distribution, Expected Improvement (EI) function has been proposed (Huang, 2006) and used in Bayesian optimization (Mockus, 1989; Jones, et al., 1998) to generate a new sampling location which can minimize response by maximizing the EI function. Fig.2 depicts EI function evaluated using the example used in Fig.1. The prediction capability of the GP model can be improved through the addition of new sampling points to the training data set. Initial training data used for GP model are collected from CAE simulations based on the Design of Experiments (DOE). To learn the distribution of response in design space, a space-filling algorithm such as full factorial design (Montgomery, 2017), Latin hyper sampling (McKay, et al., 2017), Optimal Latin hyper sampling (Ruichen, et al., 2005), and Sobol quasi-random point set (Sobol, 1967) are used for this purpose. With an effective sampling strategy using an adaptive sampling method, an accurate surrogate model can be trained with a fewer number of simulations (Gorissen, et al., 2010; Feurer, et al., 2015).

For training GP models from multibody dynamics simulations, there are two difficulties in terms of engineering. Firstly, it is required for an engineer to understand not only scalar performance value but also the waveform responses in order to analyze phenomena. The waveform response is usually discretized and handled as vector data but GP model cannot predict the vectorized response. A simple and straightforward idea to predict the vectorized response by using GP model is to train regression models as many as the number of elements of the vector data independently. The vector response can be then predicted by combining every vector element predicted by individual GP models. However, a predicted vector based on this idea can have rattling noise due to individual prediction error because there is no relationship among the individual GP model. Secondly, it is common in the study of vehicle dynamics that performance indices can be defined by the application of maximum operation to waveform response (e.g. maximum value of acceleration during time history of vehicle motion). The maximum operation can cause high nonlinearity because the maximum operation is discontinuous operation. The training data including discontinuously and high nonlinearity can make it difficult to train accurate GP models with limited computer resources. To avoid these two difficulties, in this paper, we propose a surrogate modeling method of vectorized response by using both the feature extraction method (Unsupervised learning) and the aforementioned regression model (Supervised learning). By direct prediction of the vector response, the rattling noise can be eliminated, and any discontinuous operation can be applied to the predicted waveform response.

In this paper, the feature extraction method is introduced to convert the waveform data into a set of uncorrelated scalar data. One of the methods to extract the feature mode is Singular Value Decomposition (SVD) (Eckart and Young, 1939; Kosambi. 1943). SVD is related to Principal Component Analysis (PCA) as also known as Proper Orthogonal Decomposition (POD) and Karhunen-Loeve transformation. Many researches of applications of SVD in mechanical design problems have been reported so far. Lumley (Lumley, 1967) has proposed a method to extract feature modes from the coherent turbulent flow. Cusumano, et al. (1994) and Feeny and Kappagantu (1998) have applied SVD to free vibration of the beam structure. Kayvantash et al. (2015) have proposed to construct a reduction mode from CAE results by using SVD. Once an accurate model is trained, expensive multi dynamics simulator can be replaced by the economical surrogate model. By using the model, many practical applications to vehicle dynamics to solve the inverse problem have been conducted. Siyu, et al. have proposed a method to solve the multi-disciplinary design optimization problem using 


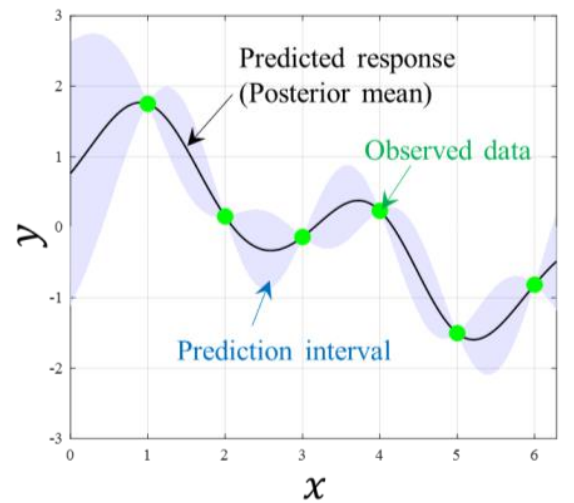

Fig.1 Demonstration of prediction of mean response and prediction interval using a GP model using a simple 1-dimensional example: $\mathrm{y}=\sin (x)+\sin (2 x)$.

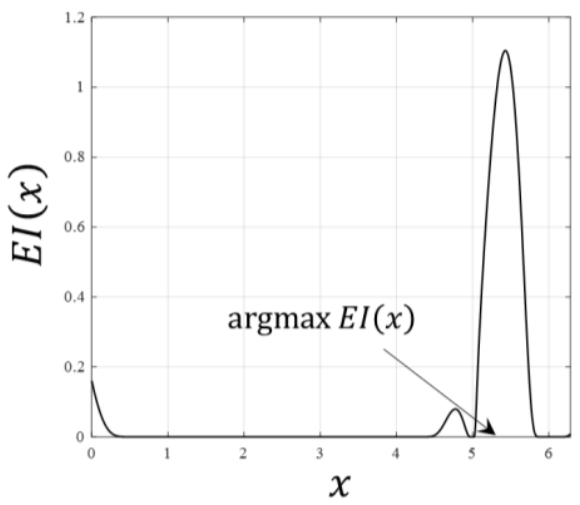

Fig.2 Expected improvement function evaluated using the example in the Fig.1. Additional design points can be obtained by maximizing the function.

trained GP model and applied it to target cascading problem of suspension design (Siyu, et al., 2018). Shintani, et al. have proposed a method to solve a feasible region using trained regression models and applied it to a target cascading problem of torsion beam type suspension (Shintani, et al., 2019). In these studies, cost functions are defined as scalar values and surrogate models to predict these cost functions are trained from simulation data. However, in this study, the prediction of transient response at the optimal solution cannot be obtained even though the optimal time series response can be required in practice.

The purpose of this paper is to propose a surrogate modeling method of waveform responses such as time series responses and frequency responses and a Bayesian optimization method using the trained waveform surrogate model. In this method, the waveform responses are decomposed into feature modes and weights by using SVD. By using SVD results, the prediction target can be converted from waveform response to uncorrelated feature weights which can be defined by projection to the feature modes. GP models of individual weight are trained. The waveform response can be predicted by the superposition of feature values calculated by GP models and feature modes. By using the posterior distribution of trained GP models, EI function is evaluated and used in Bayesian Optimization to minimize cost function estimated from the posterior mean of a predicted waveform. The feasibility of the proposed method is illustrated by application to time series response and frequency response of impact harshness phenomenon obtained by using full vehicle simulations.

\section{Feature extraction from time series responses}

In order to construct a regression model of waveform response, it is important to consider the relationship among adjacent responses. In this section, SVD is introduced and applied to decouple the relationship.

Let $i \in\{1,2, \ldots, n\}$ be an index of DOE design and $\boldsymbol{y}_{i} \in \mathbb{R}^{m}$ be $i$-th wave response vector, where $m$ is discretized number of the wave response. $\mathrm{Y}=\left\{\boldsymbol{y}_{1}, \boldsymbol{y}_{2}, \ldots, \boldsymbol{y}_{n}\right\} \in \mathbb{R}^{n \times m}$ is a training data set and can be decomposed by using SVD as:

$$
\mathrm{Y}=\mathrm{U} \Sigma \mathrm{V}^{\mathrm{T}} \text {, }
$$

where, $U=\left(\boldsymbol{u}_{1}, \boldsymbol{u}_{2}, \ldots, \boldsymbol{u}_{n}\right) \in \mathbb{R}^{n \times n}$ and $\mathrm{V}=\left(\boldsymbol{v}_{1}, \boldsymbol{v}_{2}, \ldots, \boldsymbol{v}_{m}\right) \in \mathbb{R}^{m \times m}$ are the left-singular vectors and the rightsingular vectors, respectively. The $\Sigma \in \mathbb{R}^{m \times n}$ can be written as:

$$
\Sigma=\left(\begin{array}{cc}
\mathrm{D} & 0_{\mathbb{R}^{r \times(n-r)}} \\
0_{\mathbb{R}^{(m-r) \times r}} & 0_{\mathbb{R}^{(m-r) \times(n-r)}}
\end{array}\right), \mathrm{D}=\operatorname{diag}\left(\lambda_{1}, \lambda_{2}, \ldots, \lambda_{r}\right), \quad \lambda_{1} \geq \lambda_{2} \geq \cdots, \lambda_{r} \quad \geq 0,
$$

where, $r \in \mathbb{N}$ is the rank of $\mathrm{Y}$, and $\lambda_{1}, \lambda_{2}, \ldots, \lambda_{r}$ are singular values. These singular values can be obtained by solving the following eigenvalue problem.

$$
\mathrm{Y}^{\mathrm{T}} \mathrm{Y} \boldsymbol{v}_{j}=\lambda_{j}^{2} \boldsymbol{v}_{j}
$$

Using the singular value $\lambda_{j}$ and right-singular vectors $\boldsymbol{u}_{j}$, the left-singular vectors can be obtained as: 


$$
\boldsymbol{u}_{j}=\mathrm{Y} \boldsymbol{v}_{j} / \lambda_{j}
$$

The training data $\mathrm{Y}$ can be transformed by projection to the right-singular vectors as:

$$
\mathrm{W}=\left\{\boldsymbol{w}_{1}, \boldsymbol{w}_{2}, \ldots, \boldsymbol{w}_{n}\right\}=\mathrm{YV} .
$$

Each element of $\boldsymbol{w}_{i} \in \mathbb{R}^{m}$ can be applied for the regression model independently against other elements because the right-singular vectors $\boldsymbol{v}_{i}$ is one of the orthonormal basis of $\mathbb{R}^{m}$. In this approach, regression models are trained by $m$ times, In order to save computation cost of training of GP models, the dimension of the data size $m$, can be reduced to $\bar{m}$ while satisfying the following inequality constraint.

$$
\sum_{j=1}^{\bar{m}} \lambda_{j}>\alpha \sum_{j=1}^{r} \lambda_{j}
$$

where, $\alpha \in[0,1]$ is threshold introduced to control the balance between information loss and reduced number of dimension. Using the dimensional reduction result, dimension size of $\mathrm{V}$ can be reduced as $\overline{\mathrm{V}} \in \mathbb{R}^{\bar{m} \times \bar{m}}$.

\section{Gaussian Process regression of feature value}

If feature values of $\mathrm{W}$ can be extracted from waveform responses $\mathrm{Y}$ by using SVD, it is possible to train the regression model independently of each feature value with respect to design variables, respectively because each element of training target is decoupled. In this section, we introduce GP model as follows. One advantage of choosing the GP is availability of Bayesian optimization described in a later chapter.

Let $\mathcal{D}=\left\{\left(\boldsymbol{x}_{i}, w_{i}^{j}\right)\right\}_{i=1}^{n}, j \in\{1,2, \ldots, \bar{m}\}$ be an initial training data set obtained from CAE simulation based on the space-filling DOE design, where $\boldsymbol{x}_{i} \in \mathcal{X} \subset \mathbb{R}^{d}$ be design variables. By using the GP model, $j$-th feature values $w^{j} \in \mathbb{R}$ can be approximated as:

$$
f \sim \mathcal{G P}\left(w^{j} \mid m(\boldsymbol{x}), k(\boldsymbol{x}, \dot{\boldsymbol{x}})\right),
$$

where $m: \mathcal{X} \rightarrow \mathbb{R}$ represents the mean function (polynomial or exponential). By replacing $m(\boldsymbol{x})$ with $\mu_{0} \in \mathbb{R}$, which is the global mean of given sample points. $k: \mathcal{X} \times \mathcal{X} \rightarrow \mathbb{R}$ is a kernel function, which is a positive semi-defined function. The kernel function can be written as:

$$
k(\boldsymbol{x}, \dot{x})=\sigma_{0}^{2} \mathbf{R}(\boldsymbol{x}, \dot{x})
$$

for any $\boldsymbol{x}, \dot{x} \in \mathcal{X}$, where $\mathbf{R}(\boldsymbol{x}, \dot{x})$ is the spatial correlation function and $\sigma_{0}^{2}$ is the model variance. The Gaussian kernel function, a special exponential power case for $n$-dimensional data sampling, is widely used and given by

$$
\mathbf{R}(\boldsymbol{x}, \boldsymbol{x})=\exp \left\{-\sum_{i=1}^{d} \theta_{i}\left(\boldsymbol{x}_{i}-\dot{x}_{\imath}\right)^{2}\right\}
$$

where, $\boldsymbol{\theta} \in \mathbb{R}^{n}$ is a positive scale parameter that controls the smoothness of the model.

In the prediction of weight value with respect to high order singular modes, it can be commonly assumed that response has high-nonlinearity and discontinuity. In this case, Matérn kernel is appropriate to be chosen because this function can handle high-nonlinearity and non-differentiable functions.

$$
\mathbf{R}(\boldsymbol{x}, \dot{x})=\alpha_{0} \frac{2^{1-v}}{\Gamma(v)}(\sqrt{2 v}\|\boldsymbol{x}-\dot{x}\|)^{v} K_{v}(\sqrt{2 v}\|\boldsymbol{x}-\dot{x}\|)
$$

where $\Gamma$ and $K_{v}$ are Gamma function and modified Bessel function, respectively. This kernel has two parameters $\alpha_{0}$ and $v$. The smoothness of function can be controlled by the parameter of $v$. If $v \rightarrow \infty$, The Matérn kernel is equivalent to the Gaussian kernel in Eq.(9). $\boldsymbol{\theta}, \mu_{0}, \sigma_{0}^{2}$ are hyper parameters which can be estimated via maximum likelihood estimation (MLE) (Currin, et al., 1991) as:

$$
\widehat{\boldsymbol{\theta}}, \widehat{\mu_{0}}, \widehat{\sigma_{0}^{2}}=\underset{\boldsymbol{\theta}, \mu_{0}, \sigma^{2}}{\arg \max }-\frac{n}{2} \log \left(\sigma_{0}^{2}\right)-\frac{1}{2} \log (|\mathbf{R}|)-\frac{1}{2 \sigma^{2}}\left(\boldsymbol{y}-\boldsymbol{i} \mu_{0}\right){ }^{\mathrm{T}} \mathbf{R}^{-1}\left(\boldsymbol{y}-\boldsymbol{i} \mu_{0}\right),
$$

where, $\boldsymbol{i}$ is $n$-vector of 1. Due to its logarithmic formulation, the closed-form solutions can be found as functions of $\theta$ 
as:

$$
\begin{aligned}
\widehat{\mu_{0}} & =\frac{\boldsymbol{i}^{\mathrm{T}} \mathbf{R}^{-1} \boldsymbol{y}}{\boldsymbol{i}^{\mathrm{T}} \mathbf{R}^{-1} \boldsymbol{i}^{\prime}} \\
\hat{\sigma}^{2} & =\frac{1}{n}\left(\boldsymbol{y}-\boldsymbol{i} \mu_{0}\right){ }^{\mathrm{T}} \mathbf{R}^{-1}\left(\boldsymbol{y}-\boldsymbol{i} \mu_{0}\right) .
\end{aligned}
$$

The mean value $\mu(\boldsymbol{x})$ and variance $\sigma^{2}(\boldsymbol{x})$ can be written as:

$$
\begin{aligned}
& \mu(\boldsymbol{x})=\widehat{\mu_{0}}+\boldsymbol{r}^{\mathrm{T}}(\boldsymbol{x}) \mathbf{R}^{-1} \boldsymbol{r}(\boldsymbol{x})\left(\boldsymbol{y}-\widehat{\mu_{0}} \boldsymbol{i}\right) \\
& \sigma^{2}(\boldsymbol{x})=\hat{\sigma}^{2}\left[1-\boldsymbol{r}^{\mathrm{T}}(\boldsymbol{x}) \mathbf{R}^{-1} \boldsymbol{r}(\boldsymbol{x})+\frac{\left(\mathbf{1}-\boldsymbol{i}^{\mathrm{T}} \mathbf{R}^{-1} \boldsymbol{y}\right)^{2}}{\boldsymbol{i}^{\mathrm{T}} \mathbf{R}^{-1} \boldsymbol{i}}\right],
\end{aligned}
$$

where, $\boldsymbol{r}: \mathbb{R}^{d} \rightarrow \mathbb{R}^{n}$ represents the correlation between an unknown point and all known points

$$
\boldsymbol{r}(\boldsymbol{x})=\left\{\mathbf{R}\left(\boldsymbol{x}, x_{1}\right), \mathbf{R}\left(\boldsymbol{x}, x_{2}\right), \ldots, \mathbf{R}\left(\boldsymbol{x}, x_{n}\right)\right\}^{\mathrm{T}} .
$$

In this paper, we extend the posterior information of the scalar response to waveform response. By taking a superposition of posterior distribution of GP models and the feature modes $v_{j}, j \in\{1,2, \ldots, \bar{m}\}$, mean waveform response $\boldsymbol{y}(\boldsymbol{x}) \in \mathbb{R}^{m}$, and its variance $\boldsymbol{s}^{2}(\boldsymbol{x}) \in \mathbb{R}^{m}$ can be predicted as:

$$
\begin{aligned}
& \boldsymbol{y}(\boldsymbol{x})=\sum_{j=1}^{\bar{m}} \mu_{j}(\boldsymbol{x}) \boldsymbol{v}_{j}, \\
& \boldsymbol{s}^{\mathbf{2}}(\boldsymbol{x})=\sum_{j=1}^{\bar{m}} s_{j}^{2}(\boldsymbol{x}) \boldsymbol{v}_{j} \circ \boldsymbol{v}_{j},
\end{aligned}
$$

where the $\circ$ is Hadamard product.

\section{Bayesian Optimization using posterior distribution of waveform}

Once the waveform response is predicated by using GP models, it is possible to define a cost function form waveform. Let $f: \mathbb{R}^{m} \rightarrow \mathbb{R}$ be an operator to evaluate cost function from waveform response. $f(\boldsymbol{y}(\boldsymbol{x}))$ and $f(\boldsymbol{s}(\boldsymbol{x}))$ are the posterior mean and deviation of the cost function obtained by the operator. Probability of $f(\boldsymbol{y}(\boldsymbol{x}))$ at location $\boldsymbol{x}$ being lower than a threshold $f_{0}$ can be written as:

$$
I(\boldsymbol{x})=\left\{\begin{array}{cc}
f_{0}-f(\boldsymbol{y}(\boldsymbol{x})) & \text { if } f(\boldsymbol{y}(\boldsymbol{x}))<f_{0} \\
0 & \text { otherwise }
\end{array} .\right.
$$

The expected value of Eq. (19) can be written as:

$$
E I(\boldsymbol{x})=\left(f_{0}-f(\boldsymbol{y}(\boldsymbol{x}))\right) \Phi\left(\frac{f_{0}-f(\boldsymbol{y}(\boldsymbol{x}))}{f(\boldsymbol{s}(\boldsymbol{x}))}\right)+f(\boldsymbol{s}(\boldsymbol{x})) \phi\left(\frac{f_{0}-f(\boldsymbol{y}(\boldsymbol{x}))}{f(\boldsymbol{s}(\boldsymbol{x}))}\right)
$$

where, $\Phi$ and $\phi$ are the normal cumulative distribution function and the normal probability density function, respectively. Additional sampling location which can minimize the cost function is iteratively generated by maximizing the EI function with respect to $\boldsymbol{x}$ as:

$$
\boldsymbol{x}_{n+1}=\underset{x \in \mathcal{X}}{\operatorname{argmax}} E I(\boldsymbol{x})
$$

The training data is updated as $\mathcal{D}=\left\{\left(\boldsymbol{x}_{i}, w_{i}^{j}\right)\right\}_{i=1}^{n+1}, j \in\{1,2, \ldots, \bar{m}\}$ after solving corresponding $\boldsymbol{y}_{n+1}$ and SVD results. The addition of the sampling by Eq. (21) is done iteratively until cost function doesn't change.

\section{Numerical example}

In order to show the validity of the proposed method, a numerical example of the design problem of suspension bushing with respect to the impact harshness phenomena is demonstrated in this chapter. The impact harshness is the sound and vibration when the vehicle goes over bump objects such as juncture of the road. The bump object causes 


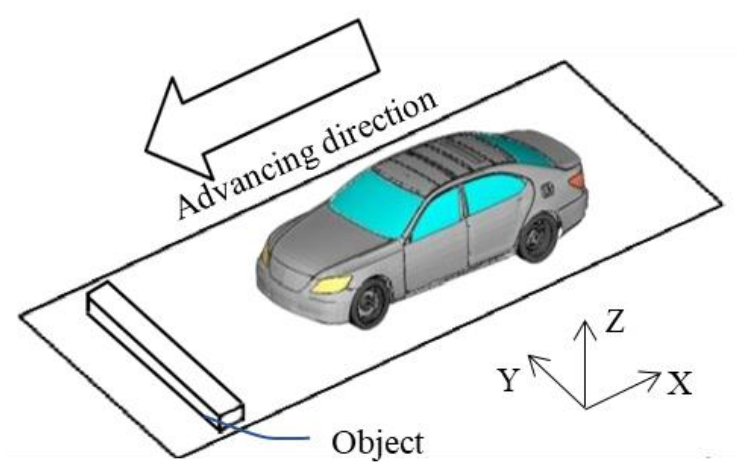

Fig.3 Condition of multi body dynamics simulation with respect to the impact harshness phenomena. A full vehicle model goes over a bump object.

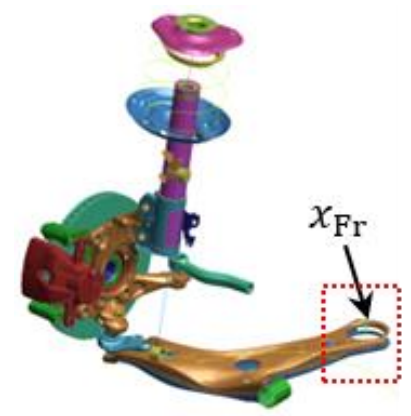

(a) Front suspension model

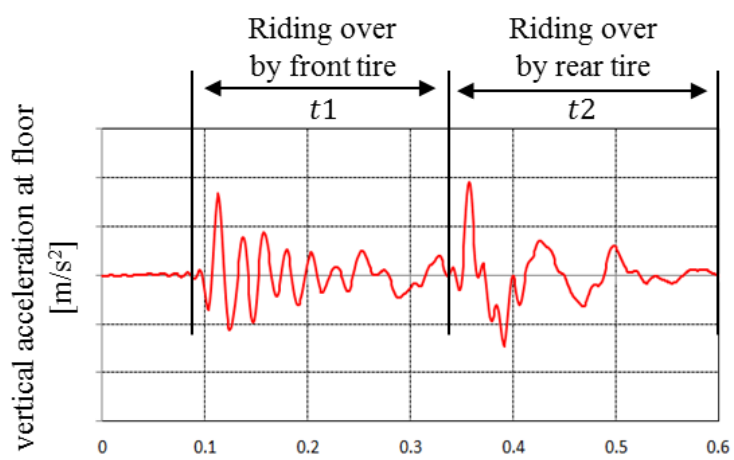

Fig.4 Impact harshness response of vertical acceleration at the floor. There are two response zones $[t 1, t 2] \in \mathrm{T}$ due to riding over by front and rear tire.

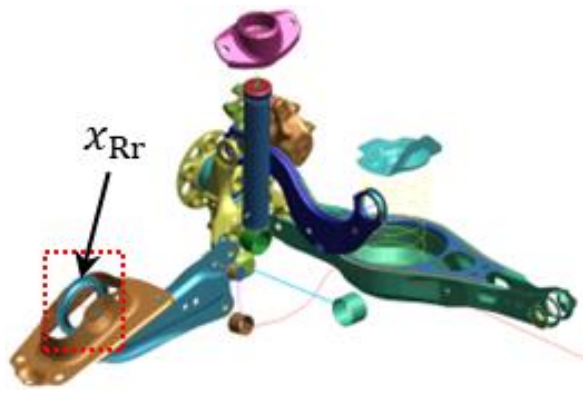

(b) Rear suspension model

Fig.5 Design variables $x_{\mathrm{Fr}}$ and $x_{\mathrm{Rr}}$. Vertical stiffnesses of front and rear bushing located at the red boxes are chosen.

Factors to these base properties are selected as design variables.

vertical and longitudinal acceleration and the driver can feel a vibration from steering, seat, and floor. Impact harshness is an important factor in ride comfort. A theoretical analysis of the impact harshness has been studied so far (Blommer, et al., 2003; Yang and Medepalli, 2005).

Fig. 3 shows a condition of multibody dynamics simulation with respect to the impact harshness. A full vehicle model in which elastic body parts reduced by using the CMS is used. Fig. 4 shows a typical time-series response of vertical accretion on the floor. There are two major response zones $[t 1, t 2] \subset \mathrm{T}$ due to riding over by front tire and rear tire. For design variables, two vertical stiffnesses from two rubber bushings are chosen in this example because the response of impact harshness is related to vertical stiffness of the front and rear suspension. For upper and lower boundary conditions, we introduce two factors to base stiffness value $x_{\mathrm{Fr}} \in[0.2,5.0]$ and $x_{\mathrm{Rr}} \in[0.2,5.0]$, respectively. Fig. 5 shows the front and rear suspension model and locations of these chosen rubber bushings are depicted by red boxes. These stiffnesses of rubber bushings have nonlinear behavior with respect to displacement because of material nonlinearly and large deformation of hyper-elastic material.

Hereafter in section 5.1, SVD is applied to the training data set of the time series response and its frequency response respectively. In section 5.2, surrogate models of these waveforms are trained using GP models and SVD results. Then, in section 5.3, an optimization problem is defined based on the trained model of the time series response and solved by using the Bayesian optimization method.

\subsection{Singular value decomposition of the waveform of impact harshness}

Based on full factorial DOE design where each design space has 11 levels, 121 designs are generated uniformly in the design space. Multibody dynamics simulations were implemented by 121 times based on the space-filling DOE designs. The 36 designs are chosen as training data for GP models and the others are used to measure accuracy. Fig.6 (a), (b) show training data set of time series response and frequency response, respectively. It can be seen from these figures 


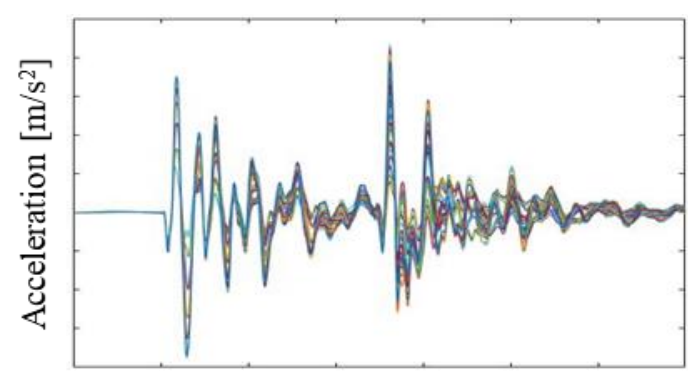

Time $[\mathrm{s}]$

(a) Time series responses

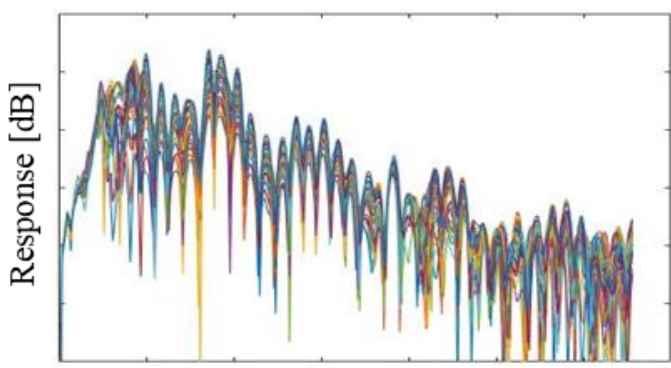

Frequency $[\mathrm{Hz}]$

Fig.6 Training data of waveform responses based on space filing DOE designs (36 designs). These waveforms can be changed dramatically by the variation of design variables.

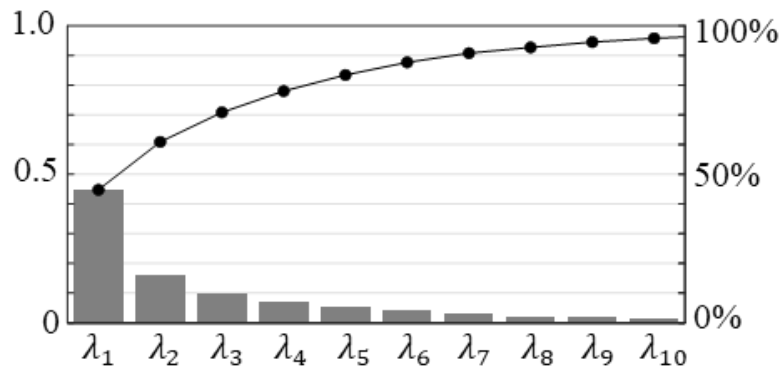

(a) Time series responses

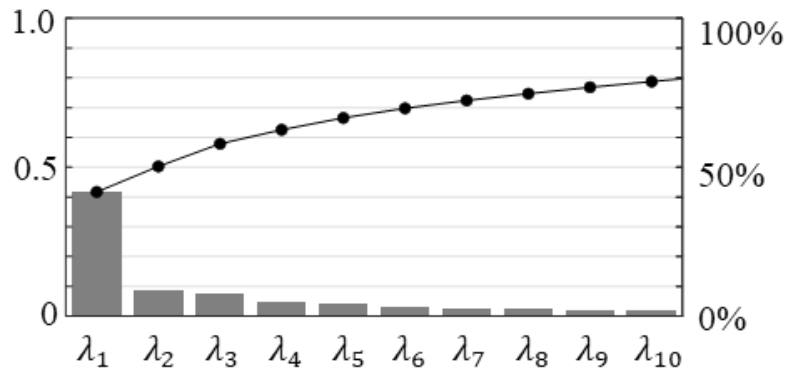

(b) Frequency responses

Fig.7 Pareto charts of first 10 dominant singular values obtained by application SVD to training data. 32 and 36 dominant feature modes are extracted to satisfy the threshold of $\alpha=0.999$ in Eq.(6).

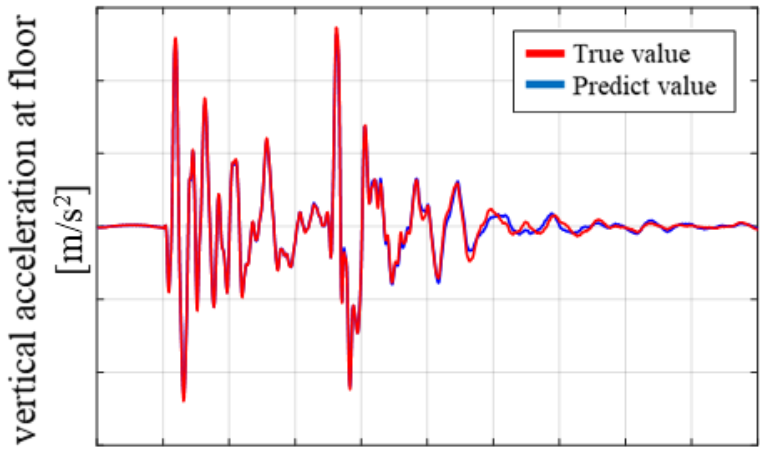

Time $[\mathrm{s}]$

(a) Comparison of Time series response

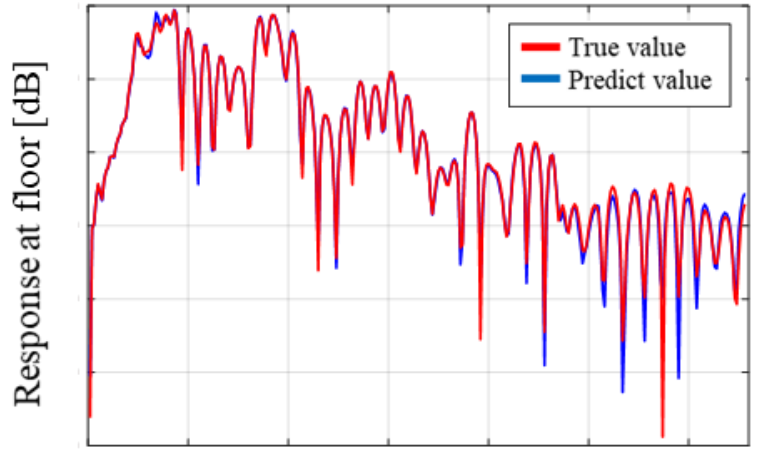

Frequency $[\mathrm{Hz}]$

(b) Comparison of frequency response

Fig.8 Comparison of time series response between predicted vector and true vector at the center point of test data $\left\{x_{\mathrm{Fr}}, x_{\mathrm{Rr}}\right\}=\{2.5,2.5\}$. accuracy of the surrogate model can be confirmed that prediction value (blue line) almost corresponds to the true value (red line).

that waveforms can be changed dramatically by the variation of design variables. To extract feature modes from these waveforms, SVD was applied. Fig.7 (a), (b) show pareto charts of the first 10 dominant singular values. In these figures, singular values are normalized. In the target setting study of rubber bushing property regarding the NVH performance, the error of the surrogate model needs to be less than $10 \%$. Therefore, setting an acceptable number of feature modes by SVD was required, considering both accuracy and computational cost. In this numerical example, a large threshold was set as $\alpha=0.999$ in Eq.(6) in order to train an accurate surrogate model, because computational cost of GP model for this numerical example is not large. As a result of the dimensional reduction by SVD, 32 and 36 dominant feature modes were extracted for time series response and frequency response, respectively. Based on the extracted feature modes, GP models were trained by 32 and 36 times, respectively. 


\subsection{Surrogate modeling of the waveform of impact harshness}

Fig.8 (a), (b) show a comparison of time series response and frequency response between the predicted value (blue line) and true value. In these figures, a test data point located at the center of design space $\left\{x_{\mathrm{Fr}}, x_{\mathrm{Rr}}\right\}=\{2.5,2.5\}$ is used. From these figure, accuracy of the trained surrogate model can be confirmed that prediction value (blue line) almost corresponds to the true value (red line). Fig.9 (a)-(d) show comparisons of prediction and true response of time series response regarding with respect to the maximum and minimum acceleration in $t 1$ and $t 2$ domain, respectively. In these figures, comparisons are implemented by using all 85 test data which is not used for training data. Fig. 10 shows

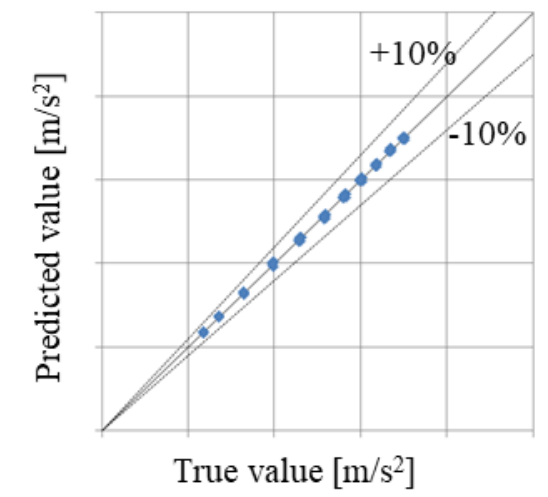

(a) The maximum value (riding over by front tire)

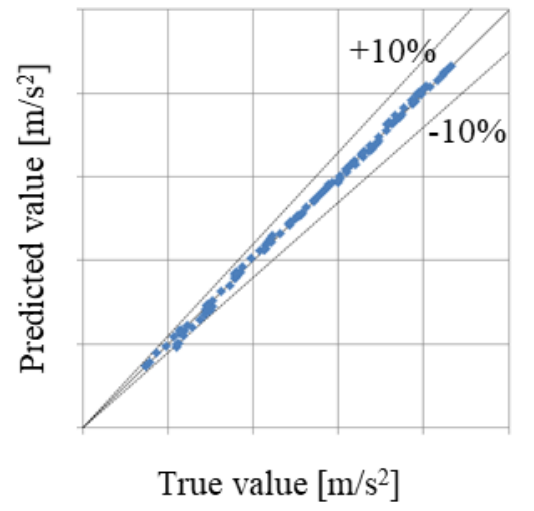

(c) The maximum value (riding over by rear tire)

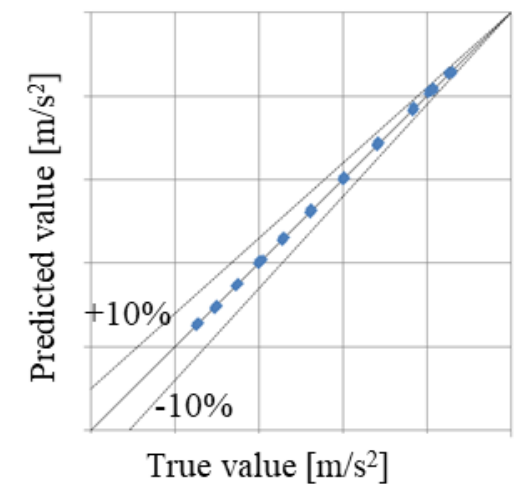

(b) The minimum value (riding over by front tire)

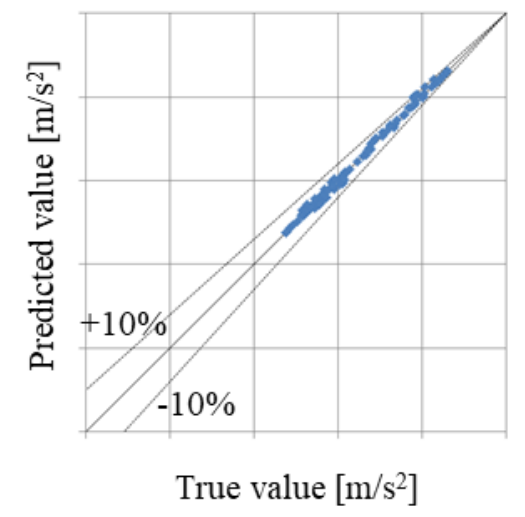

(d) The minimum value (riding over by rear tire)

Fig.9 Comparison between predict and the true value of time series response. It can be seen that predicted error is less than $10 \%$. These comparisons are implemented by using 85 test data. The trained surrogate model can replace the simulation result accurately because scattering dots in these figures are almost on the diagonal.

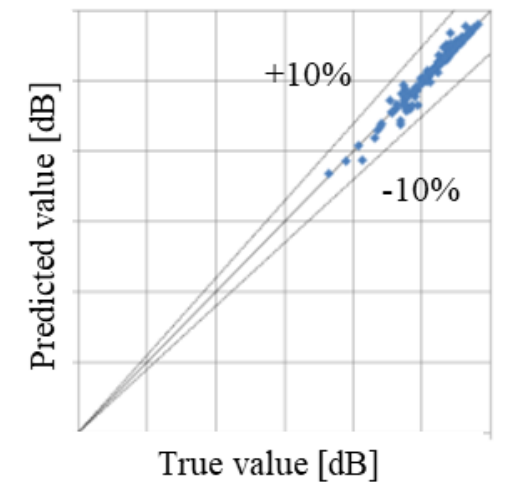

(a) The maximum value

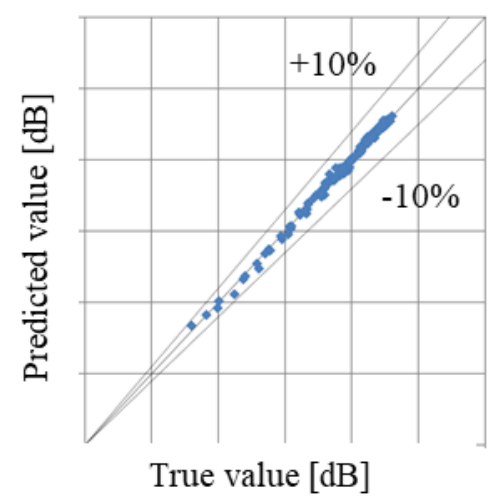

(b) The mean value

Fig.10 Comparison between predict and the true value of frequency response. It can be also seen that predicted error is less than $10 \%$. These comparisons are implemented by using 85 test data. The trained surrogate model can replace the simulation result accurately because scattering dots in these figures are almost on the diagonal. 
comparisons of prediction and true response of frequency response regarding maximum and mean value. It can be also observed that the trained surrogate model can replace the simulation result accurately because all scatter dots in these figures are almost on the diagonal.

\subsection{Bayesian optimization to minimize dominant damping ratio}

In this numerical example, a Bayesian optimization based on the posterior distribution of the proposed surrogate model is demonstrated. We introduce a cost function shown in Eq.(22)

$$
J_{0}(\boldsymbol{x})=\max \left[o_{1 s t}\left(\boldsymbol{i}_{t 1}^{\mathrm{T}} \boldsymbol{y}(\boldsymbol{x})\right) / o_{2 n d}\left(\boldsymbol{i}_{t 1}^{\mathrm{T}} \boldsymbol{y}(\boldsymbol{x})\right), o_{1 s t}\left(\boldsymbol{i}_{t 2}^{\mathrm{T}} \boldsymbol{y}(\boldsymbol{x})\right) / o_{2 n d}\left(\boldsymbol{i}_{t 2}^{\mathrm{T}} \boldsymbol{y}(\boldsymbol{x})\right)\right]
$$

In Eq.(22), $\boldsymbol{i}_{t 1}, \boldsymbol{i}_{t 2} \in \mathbb{R}^{m}$ represent a vector whose elements have 1.0 in time zone $t 1$ and $t 2$ otherwise, elements are 0.0 , respectively. The $o_{1 s t}(\cdot)$ and $o_{2 n d}(\cdot)$ are operators to extract the first largest peak and the second-largest peak from time-series response, respectively. An optimization problem to minimize the Eq.(22) is defined and solved by using Bayesian optimization as follows. This cost function means the dominant damping ratio.

For the initial design, 9 designs are generated uniformly in the design space and the surrogate model of time series response is trained using the proposed method. Among the initial training data, the smallest cost function value was observed as 0.376 at $\left\{x_{\mathrm{Fr}}, x_{\mathrm{Rr}}\right\}=\{2.50,0.20\}$. Fig. 11 shows the iteration history of Bayesian optimization. During the optimization, 11 samples are added and the cost function was reduced by $14 \%$, its optimized value was found as 0.323 at $\left\{x_{\mathrm{Fr}}, x_{\mathrm{Rr}}\right\}=\{1.56,0.20\}$. Fig.12 shows the distribution of the cost function by using the trained surrogate model. In this figure, blue dots and black dots represent the initial DOE design and the added design during Bayesian optimization, respectively. It can be seen that these 11 designs are added while balancing of sampling in underexplored areas (exploration) and sample close to already sampled points (exploitation).

In this numerical example a 2-dimentional problem was demonstrated, but the proposed method can also be expanded to high dimensional problems. However, the required number of DOE samples will be increased due to the curse of dimensionality, which causes computational burden in SVD $O\left(n^{2}\right)$ and GP $O\left(n^{3}\right)$. Therefore, for application to high dimensional problem, it is important to reduce the number of design variables by dimensional reduction or decoupling of design problem in advance.

In addition, the adaptive range of the proposed method is identical to the one of GP. In this study, GP model was chosen to train an accurate surrogate model and optimized design effectively with limited training data obtained from expensive simulations. However, if collecting a large number of samples is possible without considering the computational cost, other regression models using deep learning can be more effective in terms of computational burden and prediction accuracy.

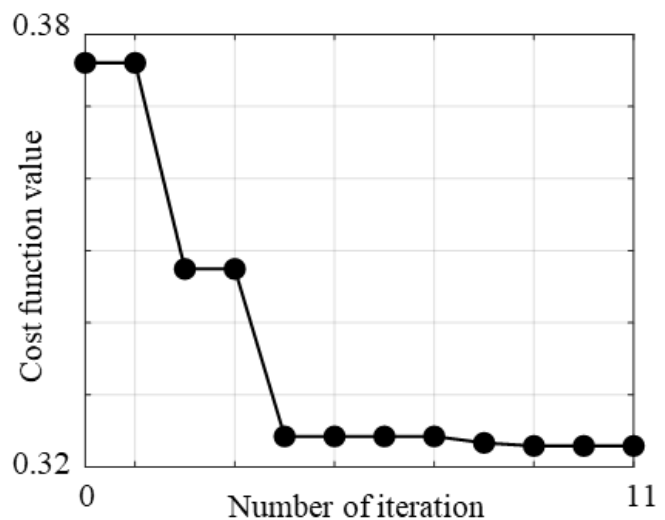

Fig.11 Iteration history of Bayesian optimization. 11 samples are added during the optimization procedure. The cost function is reduced by $14 \%$.

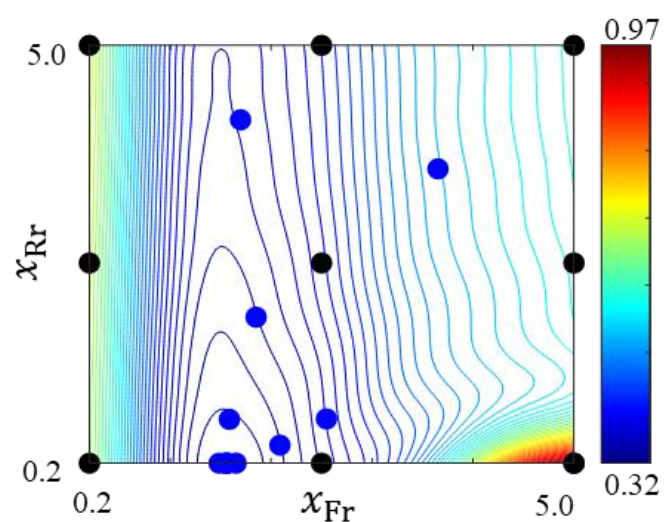

Fig.12 Distribution of cost function of Eq.(22) by using the trained surrogate model. The optimal solution was found at $\left\{x_{\mathrm{Fr}}, x_{\mathrm{Rr}}\right\}=\{1.56,0.20\}$. 


\section{Conclusion}

This paper presented a method to construct a surrogate model which can predict waveform response and a solution of Bayesian optimization using posterior distribution of trained waveform response. In this proposed method, supervised and unsupervised learning were introduced. For unsupervised learning, the singular value decomposition was used as a feature extraction method and applied to the training data. Obtained singular vectors were used as feature modes to represent the training data of waveform. For unsupervised learning, Gaussian Process was introduced as a regression model and applied to each weight of the feature modes which can be obtained by projection of training data to the feature modes. The waveform response was predicted by the superposition of predicted feature values and the feature modes. By using the trained Gaussian process models, Expected Improvement function with respect to cost function defined based on the posterior distribution of waveform response was evaluated. Bayesian Optimization was introduced to minimize the cost using the Expected Improvement function. The feasibility of the proposed method was illustrated by application to a suspension design problem of impact harshness phenomenon by using full vehicle simulation. In the numerical example, it was confirmed that accurate surrogate models of both time series response and frequency response were obtained. Moreover, an optimization problem to minimize the dominant damping ratio by using the trained surrogate model was solved effectively by using Bayesian optimization method.

\section{References}

Blommer, M., Amman, S., Gu, P., Tsou, P., Dawson, V., and Vandenbrink, K.. Sound Quality Aspects of Impact Harshness for Light Trucks and SUVs, SAE Technical Paper, No. 2003-01-1501 (2003).

C. E. Rasmussen and C. K. Williams, Gaussian Processes for Machine Learning. University Press Group Limited, 2006.

Currin, C., Mitchell, T., Morris, M. and Ylvisaker, D., Bayesian prediction of deterministic functions with applications to the design and analysis of computer experiments, Journal of the American Statistical Association86, No. 416 (1991), pp. 953-963.

Cusumano, J. P., Sharkady, M. T. and Kimble, B. W., Experimental measurements of dimensionality and spatial coherence in the dynamics of a flexible-beam impact oscillator, Philosophical Transactions of the Royal Society of London. Series A: Physical and Engineering Sciences 347, No. 1683 (1994), pp. 421-438.

Eckart, C., and Young, G., A principal axis transformation for non-Hermitian matrices, Bulletin of the American Mathematical Society 45, No. 2 (1939), pp.118-121.

Feurer, M., Klein, A., Eggensperger, K., Springenberg, J., Blum, M. and Hutter, F., Efficient and robust automated machine learning, Proceedings of the Advances in neural information processing systems, (2015), pp. 2962-2970.

Fonseca, D. J., Navaresse, D. O., \& Moynihan, G. P, Simulation metamodeling through artificial neural networks, Engineering Applications of Artificial Intelligence, Vol. 16, No. 3 (2003), pp. 177-183.

Gillespie, T. D., Fundamentals of vehicle dynamics, SAE Technical Paper, Vol. 114. (1992).

Gorissen, D., Grid-enabled adaptive surrogate modeling for computer aided engineering, Ph.D. Thesis, Dept. of Computer Science, Ghent University, (2010).

Gorissen, D., Couckuyt, I., Demeester, P., Dhaene, T. and Crombecq, K., A surrogate modeling and adaptive sampling toolbox for computer based design, Journal of Machine Learning Research, No.11 (2010), pp. 2051-2055.

Huang, D., Allen, T. T., Notz, W. I. and Miller, R. A, Sequential kriging optimization using multiple-fidelity evaluations, Structural and Multidisciplinary Optimization,Vol. 32, No. 5 (2006), pp. 369-382.

Hutter, F., Hoos, H. H., and Leyton-Brown, K., Sequential model-based optimization for general algorithm configuration, Proceedings of the International conference on learning and intelligent optimization, (2011), pp. 507523

Hintz, R. M., Analytical methods in component modal synthesis, AIAA Journal, Vol. 13, No. 8 (1975), pp.1007-1016.

Jones, D. R., Schonlau, M., and Welch, W. J., "Efficient global optimization of expensive black-box functions.”, Journal of Global Optimization, Vol.13, No.4 (1998), pp.455-492.

Jin, Ruichen, Wei Chen, and Agus Sudjianto., An efficient algorithm for constructing optimal design of computer experiments, Journal of statistical planning and inference Vol.134, No.1 (2005), pp. 268-287. 
Kayvantash, K., Thiam, A. T., Ryckelynck, D., Chaabane, S. B., Touzeau, J. and Ravier, P, Model Reduction Techniques for LS-DYNA ALE and Crash Applications, In Proceedings of 10th European LS-DYNA Conference, (2015).

Kosambi, D. D., Statistics in function space, Journal of the Indian Mathematical Society, No.7 (1943), pp. 76-88.

Lumley, J. L., The structure of inhomogeneous turbulent flows, In Proceedings of Atmospheric Turbulence and Radio Wave Propagation, (1967), pp. 166-178.

McKay, M.D., Beckman, R.J. and Conover, W.J., A comparison of three methods for selecting values of input variables in the analysis of output from a computer code, Technometrics, Vol.21, No.2 (1979), pp.239-45.

Mockus, J., Bayesian Approach to Global Optimization. Kluwer Academic Publishers, Dordrecht, (1989).

Montgomery, Douglas C. Design and analysis of experiments. John wiley \& sons, (2017).

Shintani, K., Atsuji A. and Yasushi Y., A Target Cascading Method Using Model Based Simulation in Early Stage of Vehicle Development, SAE Technical Paper, No. 2019-01-0836, (2019).

Sobol', I. Y. M., On the distribution of points in a cube and the approximate evaluation of integrals. Zhurnal Vychislitel'noi Matematiki i Matematicheskoi Fiziki, Vol.7, No.4 (1967), pp.784-802.

Suykens, J. A., and Vandewalle, J., Least squares support vector machine classifiers. Neural processing letters, Vol.9, No.3 (1999), pp.293-300.

Tao, S., Shintani, K., Yang, G., Meingast, H., Apley, D. W., and Chen, W., Enhanced Collaborative Optimization Using Alternating Direction Method of Multipliers, Structural and Multidisciplinary Optimization, Vol. 58, No. 4 (2018), pp.1571-1588.

Williams, C. K., and Rasmussen, C. E., Gaussian processes for machine learning, Cambridge, MA: MIT press, Vol. 2, No. 3 (2006).

Yang, X. and Medepalli, S., Sensitivities of suspension bushings on vehicle impact harshness performances, SAE transactions (2005), pp.438-444.

Zăvoianu, A. C., Bramerdorfer, G., Lughofer, E., Silber, S., Amrhein, W., and Klement, E. P., Hybridization of multiobjective evolutionary algorithms and artificial neural networks for optimizing the performance of electrical drives. Engineering Applications of Artificial Intelligence, Vol.26, No.8 (2013), pp.1781-1794.

Zăvoianu, A. C., Lughofer, E., Koppelstätter, W., Weidenholzer, G., Amrhein, W., and Klement, E. P., Performance comparison of generational and steady-state asynchronous multi-objective evolutionary algorithms for computationally-intensive problems. Knowledge-Based Systems, Vol.87 (2015), pp.47-60. 